\title{
Recent results from low- $x$ and forward physics at HERA
}

\author{
Lidia Goerlich on behalf of the H1 Collaboration \\ Institute of Nuclear Physics PAN, Radzikowskiego 152, 31 -342 Kraków, Poland
}

\begin{abstract}
The production of forward jets in inclusive as well as diffractive deep inelastic scattering at HERA is studied with the $\mathrm{H} 1$ detector.

For inclusive DIS events at low $Q^{2}$ with a forward jet, produced close to the proton remnant, differential cross sections and normalised distributions are measured as a function of the azimuthal angle difference between the forward jet and the scattered positron. Results on dijet production cross sections are also presented for diffractive DIS events in which the final state proton is tagged in the H1 Forward Proton Spectrometer. Two topologies are investigated by either requiring the two jets being produced centrally or by requiring that one of the jets is going in the forward direction.

The data are compared with NLO QCD predictions as well as leading order Monte Carlo models.
\end{abstract}

Keywords: deep inelastic scattering, Quantum Chromodynamics, diffraction, low- $x$, jets PACS: $13.60 . \mathrm{Hb}$

\section{INTRODUCTION}

The HERA ep collider has extended the available kinematic range for deep-inelastic scattering (DIS) to regions of the Bjorken scaling variable, $x$, as small as $10^{-5}$ at moderate photon virtuality, $Q^{2}$, of a few $\mathrm{GeV}^{2}$. At low $x$ the quark struck by the photon may originate from a QCD cascade initiated by a parton in the proton. Several perturbative QCD-based approaches are available to describe the dynamics of the parton evolution process. In the standard DGLAP evolution [1] partons emitted in the cascade are strongly ordered in transverse momentum, $k_{T}$, measured with respect to the proton direction. At small values of $x$ a transition is expected from DGLAP to BFKL dynamics [2] in which there is no ordering in $k_{T}$ of the partons along the ladder. Measurements of DIS events with energetic jets of high transverse momentum produced close to the proton direction in the laboratory frame, referred to as the forward region, are considered to be especially sensitive to the QCD dynamics at low $x$ [3]. In this talk the study of the azimuthal correlation between the forward jet and the scattered positron in DIS is presented [4].

The observation that roughly $10 \%$ of DIS events at HERA have a gap in particle emission in a large interval of pseudorapidity around the proton remnant direction was quite a surprise. These events are called diffractive and are usually described by an exchange of a colourless object, called Pomeron. Measurements of diffractive DIS (DDIS) allow deeper insight into the QCD dynamics at low $x$.

In addition to standard DIS variables, $Q^{2}$ and $x$, the kinematics of DDIS is described by: $x_{\mathbb{P}}$, which is the fractional momentum of the proton carried by the Pomeron, $t$, which is the squared four-momentum transfer at the proton vertex and $\beta=x / x_{\mathbb{P}}$, which is the 
fractional momentum of the Pomeron carried by the struck parton. The study of DDIS process with two jets and the leading final state proton detected in the H1 Forward Proton Spectrometer is presented [5].

\section{FORWARD JET AZIMUTHAL CORRELATIONS}

The analysis phase space is restricted in $Q^{2}, x$ and inelasticty $y: 5<Q^{2}<85 \mathrm{GeV}^{2}$, $0.0001<x<0.004,0.1<y<0.7$. Events with at least one forward jet satisfying the following cuts in the laboratory frame are selected: $P_{\mathrm{T} \text {,fwdjet }}>6 \mathrm{GeV}, 1.73<$ $\eta_{\text {fwdjet }}<2.79, x_{\text {fwdjet }}=E_{\text {fwdjet }} / E_{p}>0.035$ and $0.5<P_{\mathrm{T}, \mathrm{fwdjet}}^{2} / Q^{2}<6$. Here $\eta_{\text {fwdjet }}$ is the pseudorapidity of the forward jet. The last two cuts aim to enhance the effects of BFKL dynamics and suppress the DGLAP evolution.

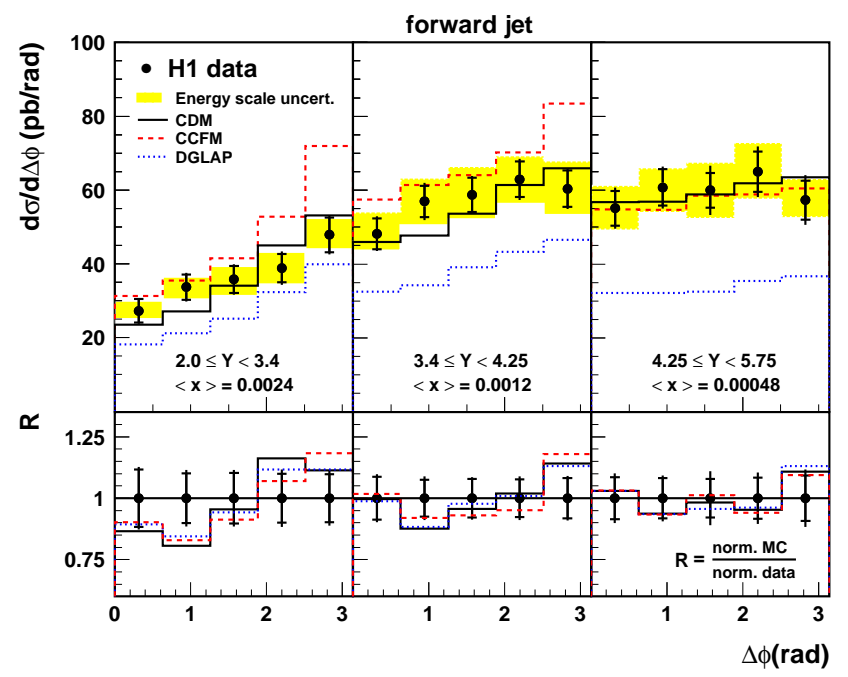

FIGURE 1. Forward jet cross section $d \sigma / d \Delta \phi$ compared with the predictions of QCD-based models.

The forward jet cross section $d \sigma / d \Delta \phi$ as a function of the azimuthal angle difference $\Delta \phi$ between the most forward jet and the scattered positron is shown in Figure 1 for three intervals of the positron-jet rapidity distance $Y$. At higher values of $Y$ the forward jet is more decorrelated from the scattered positron. The predictions of three QCDbased models with different underlying parton dynamics are compared with the data. The cross sections are well described in shape and normalisation by the BFKL-like Colour Dipole Model (CDM) [6]. Predictions based on DGLAP evolution, fall below the data, particularly at large $Y$ corresponding to low values of $x$. Calculations in the CCFM scheme [7] which unifies the DGLAP and BFKL approaches overestimate the measured cross section for large $\Delta \phi$ values in the two lowest $Y$ intervals. However, this model provides as good a description of the data as CDM in the highest $Y$ interval.

The ratio $R$ of MC to data for normalised cross sections shows that the shape of the $\Delta \phi$ distributions does not discriminate further between different evolution schemes.

NLO DGLAP predictions of the NLOJET++ program [8] shown in Figure 2 are in general below the data, but still in agreement within the large theoretical uncertainties 
indicating that in this phase space region higher order contributions are expected to be important.

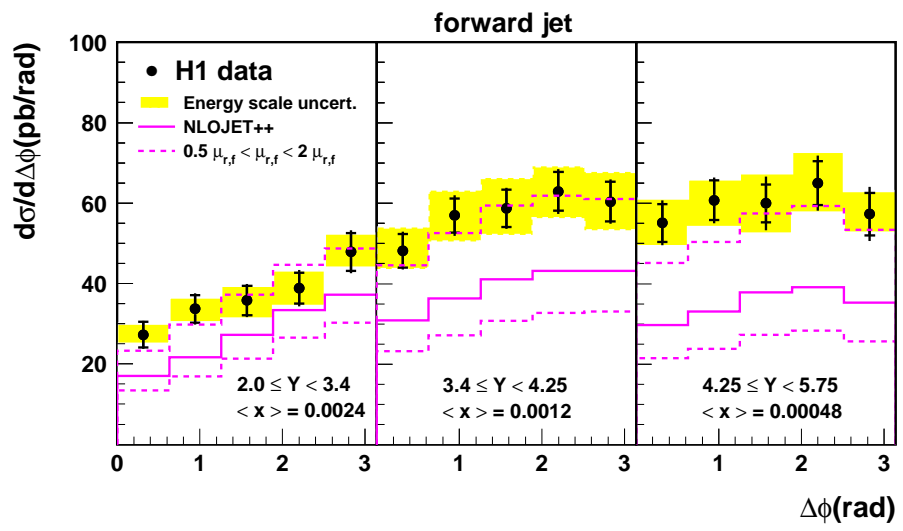

FIGURE 2. Forward jet cross section as a function of $\Delta \phi$ compared to NLO QCD predictions.

\section{DIFFRACTIVE DIJET PRODUCTION}

Differential cross sections are measured for dijet production in the DDIS process $e p \rightarrow$ $e j j X^{\prime} p$. For DDIS processes the hard scattering QCD collinear factorisation theorem allows a definition of diffractive parton distribution functions (DPDFs) and their $Q^{2}$ evolution using the DGLAP equations. Diffractive dijet production is used to study the universality of DPDFs. The measurement is performed for $x_{\mathbb{P}}<0.1$ and covers the range $0.1<|t|<0.7 \mathrm{GeV}^{2}$ and $4<Q^{2}<110 \mathrm{GeV}^{2}$. Two event topologies are investigated: a topology where two jets are found in the central pseudorapidity range and a topology where one jet is central and one jet is more forward.

Two central jets. Events with at least two jets within the pseudorapidity range $-1<\eta_{1,2}<2.5$ in the laboratory frame and transverse momenta $P_{\mathrm{T}, 1}^{*}>5 \mathrm{GeV}$ and $P_{\mathrm{T}, 2}^{*}>4 \mathrm{GeV}$ in the virtual photon-proton centre-of-mass system are selected.
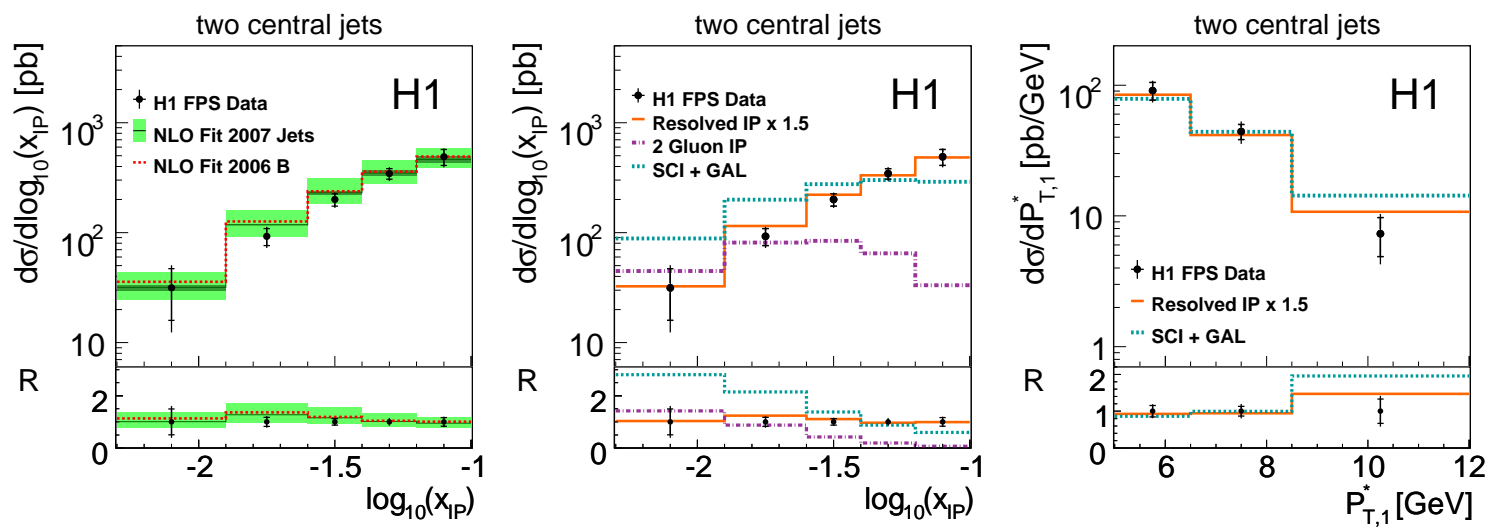

FIGURE 3. The cross section for the production of two central jets as a function of $\log _{10}\left(x_{\mathbb{P}}\right)$ and $P_{\mathrm{T}, 1}^{*}$. 
The cross sections are shown as a function of $\log _{10}\left(x_{\mathbb{P}}\right)$ and $P_{\mathrm{T}, 1}^{*}$ in Figure 3 . NLO QCD predictions based on the DGLAP approach and using the DPDFs [9] extracted from inclusive diffractive measurements describe the data within errors, supporting the universality of DPDFs. The Resolved Pomeron model [10] describes the shape of the cross sections well, but is too low in normalisation. This suggests that contributions from higher order processes are expected to be important in this approach. The Soft Colour Interaction model [11], SCI+GAL, and Two Gluon Pomeron model [12] fail to describe the shape of the distributions of the diffractive variables. However, the SCI+GAL model reproduces reasonably well the measurements as a function of the jet variable $P_{\mathrm{T}, 1}^{*}$.

One central + one forward jet. At least one central jet with $-1<\eta_{\mathrm{c}}<2.5$ and one forward jet with $1<\eta_{\mathrm{f}}<2.8$, where $\eta_{\mathrm{f}}>\eta_{\mathrm{c}}$, are required with $P_{\mathrm{T}}^{*}>3.5 \mathrm{GeV}$. Events with one central and one forward jet are used to search for effects beyond the standard DGLAP evolution. Measurements as a function of the fractional momentum of the Pomeron carried by the parton entering the hard scatter, $z \mathbb{P}$, and the azimuthal angle difference $\left|\Delta \phi^{*}\right|$ between the two jets in the $\gamma^{*} p$ frame are shown in Figure 4. The NLO DGLAP predictions also describe this event topology. The shape of the cross section as a function of $z_{\mathbb{P}}$ is well described only by the RP model.
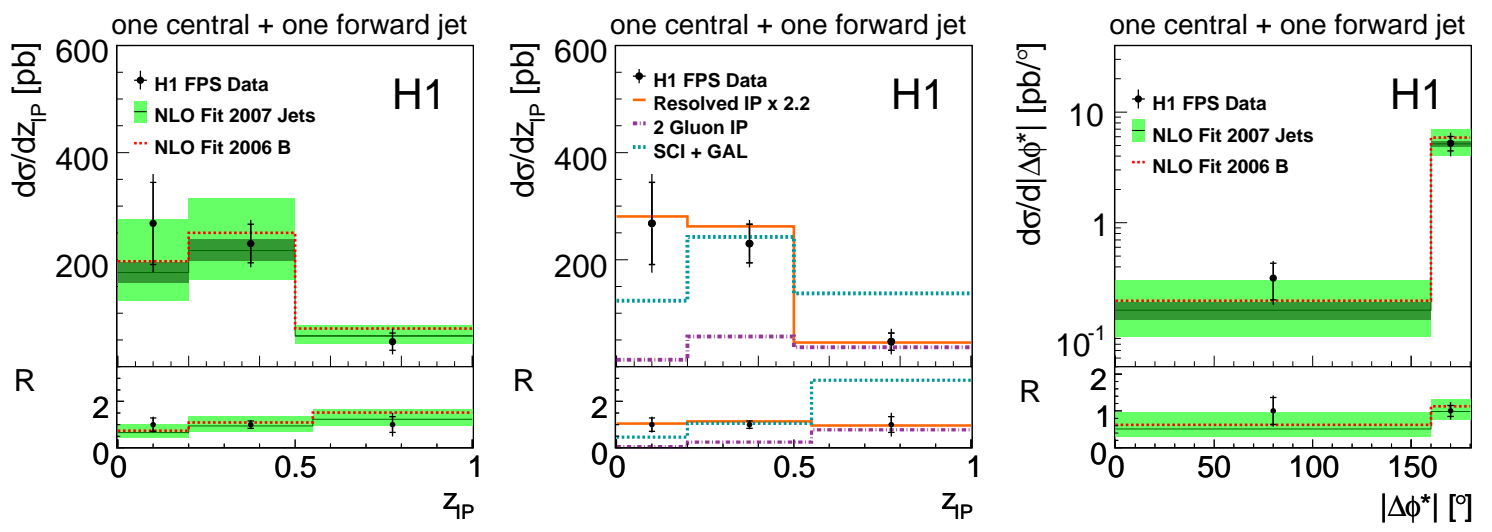

FIGURE 4. The cross section for the production of one central and one forward jet vs. $z \mathbb{P}$ and $\left|\Delta \phi^{*}\right|$.

\section{REFERENCES}

1. G. Altarelli and G. Parisi. Nucl. Phys. B 126, 298 (1977).

2. E. Kuraev, L. Lipatov and V. Fadin. Sov. Phys. JETP 45, 199 (1977).

3. A. H. Mueller. Nucl. Phys. B, Proc. Suppl. 18C, 125 (1990).

4. F. D. Aaron et al. [H1 Collaboration], Eur. Phys. J. C 72, 1910 (2012), arXiv:1111.4227.

5. F. D. Aaron et al. [H1 Collaboration], Eur. Phys. J. C 72, 1970 (2012), arXiv:1111.0584.

6. L. Lönnblad, Comput. Phys. Commun. 71, 15 (1992).

7. S. Catani, and F. Fiorani, and G. Marchesini, Nucl. Phys. B 336, 18 (1990).

8. Z. Nagy, and Z. Trocsanyi, Phys. Lett. 87, 82001 (2001).

9. A. Aktas et al. [H1 Collaboration], Eur. Phys. J. C 48, 715 (2006), arXiv:hep-ph/0606004.

10. G. Ingelman, and P. E. Schlein, Phys. Lett. B 152, 256 (1985).

11. A. Edin, and G. Ingelman, and J. Rathsman, Z. Phys. C 75, 57 (1997), arXiv:hep-ph/9605281.

12. J. Bartels et al., Phys. Lett. B 379, 239 (1996). 